INTERNATIONAL HIGHER EDUCATION, NO. 67, Spring, 2012

Pages 26-27

\title{
A New Approach for Classifying Chilean Universities
}

\author{
Claudia Reyes and Pedro Rosso
}

Claudia Reyes is executive director of Red Universitaria Cruz del Sur, in Santiago, Chile; Pedro Rosso is rector emeritus and professor of pediatrics at Pontificia Universidad Católica de Chile, in Santiago, Chile. E-mail, Claudia Reyes and Pedro Rosso: barriga@uc.cl.

Over the last few decades, particularly during the 1980s and 1990s, most university systems in the developing world underwent an impressive transformation-with several fold increases in the number of students enrolled and the opening of many new, mostly private, universities. One of the consequences of this expansive change has been a marked increase in the heterogeneity of the institutions comprised in the various systems. Beyond its academic dimensions, heterogeneity poses serious problems to systems attempting to classify the universities for research, ranking or public policies purposes. Chile is a good example. The first attempt to classify national universities — based on selectivity, size, prestige, and nature (public or private)— resulted in eight categories. Despite some of its merits, this classification was criticized on conceptual and practical grounds, including the fact that the categories were not exclusive ones.

Other observers have tried to classify Chilean universities, using selectivity and annual publications as primary criteria, and the number of 
students and the years of accreditation granted to the institution as secondary criteria. They described seven categories of institutions-some improvements over the previous ones. However, this classification was also flawed on several accounts, including the use of selectivity as a main criterion. For example, one category listed selective research universities, while another group was desribed as nonselective, teaching, large-size, and low accreditation institutions.

\section{A New ApProach to Classifying UniVersities}

A recent approach faced the challenge of classifying Chilean universities-using as main criteria the existence and number of accredited doctoral programs and the annual number of internationally indexed publications. Applying the first criterion, the universities were divided into two groups: (a) without accredited doctoral programs; and (b) with doctoral programs. Then, those without doctoral programs were subsequently divided, according to the number of publications, in two categories: (a) with less than 20 annual publications; and (b) with 20 or more annual publications. The first category was named "teaching university" and comprised 23 institutions. The second one, called "teaching university with limited research," included 11 universities. In turn, the universities with accredited PhD programs were divided in two categories: (a) those with up to five programs, and $(b)$ those with more than five doctoral programs. The first category was called "university with research and doctoral programs in selected areas," and 11 institutions met this criterion. The second one was named "research and doctoral programs university" and comprised 6 universities. 


\section{Main Characteristics of THE Different CATEgories}

As expected, the four categories had marked differences in the mean values of the variables used as "primary classification criteria." Thus, the teaching university group averaged 4 publications per year, the teaching university with research projection group averaged 41 publications per year; the "university with research and doctoral programs in selected areas" group averaged 94 annual publications; and the "research and doctoral programs university" group averaged 636 publications per year. In turn, while the average number of doctoral programs was 2.2 in the group of "university with research and doctoral programs in selected areas," it averaged 18.5 in the group of "research and doctoral programs university." "Consequently, the primary classification criteria had successfully grouped Chilean universities in markedly different categories. Particularly striking was the tenfold difference in the number of publications observed, between the two "teaching universities" categories-indicating that on this aspect the category "teaching university" is indeed quite different than its "teaching university with research projection" partner. On the other hand, this difference implies that in approximately 30 percent of the Chilean universities practically no research is conducted.

\section{Additional Characteristics With the Four Categories}

The four categories were also compared on the values of institutional size and academic performance (accreditation)-unrelated to the publications and doctoral programs indicators used to define the four categories. The statistical significance of variations in mean values between categories was tested using a 
one-way analysis of variance. This test provides a method to establish whether or not the means of several groups are statistically different. The analysis of variance test was complemented with post hoc tests, which do establish more specifically means that were significantly different, from each other. Results indicated a major diversity in mean values in most of the indicators explored, including: number of students, number of faculties, percentage of faculties with advanced degrees, number of faculties per study program, percentage of accredited study programs, and years of institutional accreditation. The main differentiations were found between the "teaching university" and the "research and doctoral programs university" categories, with mean values of the other two categories falling in between.

\section{New Classification for Comparative Studies}

The categories defined by the new classification are associated with basic institutional characteristics and academic performances. Thus, for comparison purposes, the institutions included within a given category could be considered to be "academic peers." The latter seems a relevant point, since most of the available comparative studies_-including national and international university rankings-generally overlook this aspect. From this perspective, it is unfortunate that the research universities, especially those considered to be "world class" have become the paradigm of academic quality. While recognizing the need for any country to have a "critical mass" of those institutions, from the stand point of diversity and their intrinsic value, the only paradigm that a university should have is the best institution within its own category. 


\section{Classifying Universities IN DEVEloping Systems}

The new classification used for Chilean universities can be applied in other countries, with some adaptation to local realities. For example, other cut-off points for annual publications or number of doctoral programs accredited by a national agency could be used. The new classification also might provide an overall diagnosis of a system, in terms of the percentage of teaching and research institutions present. In university systems diversity represents a value in itself, since it implies both for the students and the faculty more options to decide where to study or work.

When classifying and comparing universities, particularly in developing systems, all classifications do freeze in time essentially dynamic situations. In the future, many institutions will reform their category as research activities expand and new postgraduate programs are created. On the other hand, faithful to their missions, many other universities will remain in the same category, while improving their academic performance. Ultimately, in the academic world what really counts is coherence between mission, human, and financial resources and the will to achieve the highest possible quality standards. Thus, it is crucial to properly classify universities. 